\title{
Synthesis of Fault Tolerant Neural Networks
}

\author{
Dhananjay S. Phatak and Elko Tchernev
}

\begin{abstract}
This paper evaluates different strategies for enhancing (partial) fault tolerance (PFT) of feedforward artificial neural networks (ANNs). We evaluate a continuum of strategies between the two extremes

(i) Replicating a minimal seed network to achieve a final desired size with the resultant PFT and

(ii) Starting with the desired (larger) size but using modified training algorithms to achieve higher PFT (without any replications)

The idea is not to replicate the minimal network but somewhat larger-than-minimal network and evaluate the effect on the PFT of the resulting network. In other words we investigate the optimal size of the seed network (which gets replicated) that achieves the highest PFT for a fixed final size (i.e., the total number of units and connections).

The data demonstrate that replicating larger-than minimal networks yields higher PFT for the same final size (as compared with either replicating the minimal network or starting off with the final target size and employing modified training but not using replications at all). Furthermore, it is seen that when the size of the seed network exceeds some threshold, the PFT for a given final size typically worsens. Thus, there is an optimal size for the seed network. We provide qualitative explanation of this and allied phenomena.
\end{abstract}

\section{Introduction and Related Work}

Fault tolerance of ANNs has been investigated by many researchers (a sampling can be found in 1], [2], [3], [4], [5], [6], [7], [8], [9], [10], [11], [12], [13]). Our prior work [6], [8], [10] related fault tolerance to the amount redundancy required to achieve it. It was demonstrated that for the standard/typical ANN architectures wherein the units (or neurons) perform a weighted sum (of inputs from other units) followed by a monotonic nonlinear squashing to generate their outputs; less than TMR (Triple Modular Redundancy) is not sufficient to achieve complete fault tolerance. A simple method of replicating a seed network was shown to be one possible way of enhancing fault tolerance of ANNs. This method requires a large amount of

CSEE Dept. University of Maryland Baltimore County (UMBC), 1000 Hilltop Circle, Baltimore, MD 21250.

Email: \{phatak,etcher1\}@umbc.edu

This work was supported in part by NIST and NSF grant ECS-9875705 redundancy even if the size of the seed network (which gets replicated) is kept minimal. A better alternative might be to use enhanced training algorithms that target fault tolerance. This was analyzed in [8], [12]. The data and analysis revealed that using permuted samples and/or cross validation during training do not yield a discernible PFT enhancement. Providing initial redundancy and modifying the training algorithm/method to utilize the extra parameters does improve the partial fault tolerance (PFT) to some extent. However, a brute force method of replications proposed in [6], [10] seems to achieve a higher PFT for the same level of redundancy (as compared with the fault tolerant gradient descent training) which was a counter-intuitive result. The plausible explanation draws an analogy with the divide and conquer technique: Instead of starting off with a large number of parameters and trying to adjust all of them simultaneously, it is better to work with the least possible number of parameters, train the smallest net and then replicate it.

In principle, the configuration obtained by duplicating (replicating in general) the hidden units is just a special case of a bigger, more general net having double the number of units (more precisely, independent parameters). If one starts with a net with double the number of units (parameters), what is the probability that the training algorithm will converge to a solution that was obtained by replicating the smaller net? (this is somewhat analogous to the probability of both roots of a quadratic being identical) The probability of the search algorithm converging to such a specific solution is negligible. The probability is zero in a measure theoretic sense because the larger network has many more (twice as many) dimensions, since each independently adjustable weight represents a dimension in the search space.

In this paper we experimentally investigate the optimal size of the seed network which yields the highest PFT. The data indicate that the optimal size for a seed network is larger than minimum which is somewhat counter intuitive. We provide a qualitative explanation for this phenomenon.

\section{Experimental Setup}

We conducted experiments with the two classdistinction problem illustrated in Figure 1 and the Sonar benchmark [14], [15]. The reason for selecting these tasks is that for both these problems, the minimal sized net- 


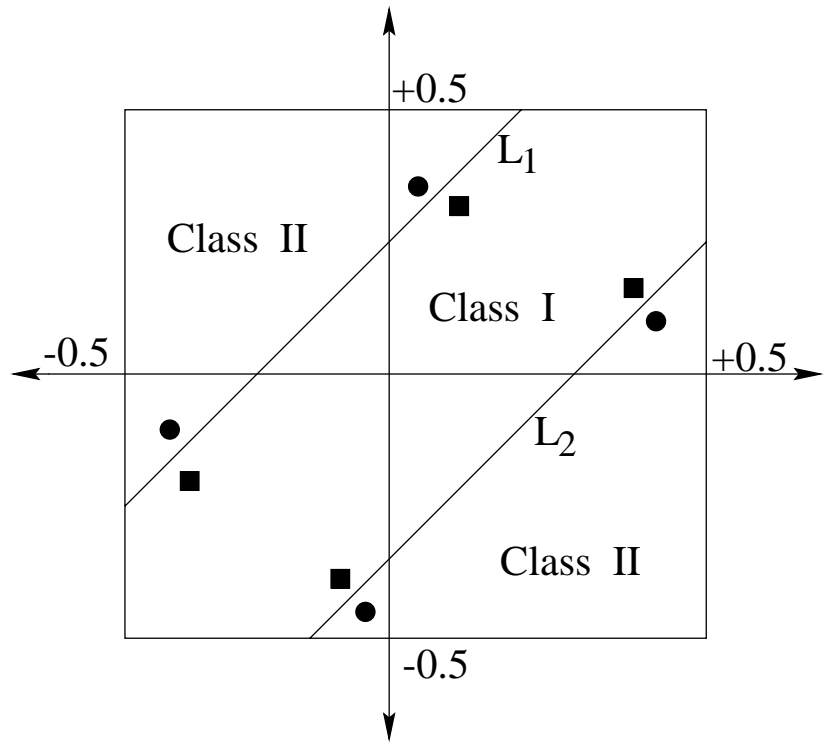

Fig. 1

THE TWO-CLASS DISTINCTION PROBLEM: DATA POINTS ARE UNIFORMLY DISTRIBUTED WITHIN THE SQUARE, BOTH CLASSES OCCUPY EQUAL AREAS. EACH TRAINING/TEST PATTERN CONSISTS OF THE $(x, y)$ COORDINATES OF THE POINT AND THE CLASS.

work (which can successfully learn to classify) is already known. Assuming a strictly layered structure (wherein, all units in a layer connect to only the units in the next adjacent layer, no layer-skipping connections to other units are present), the minimal sized network for the two-class distinction problem has two hidden units in a single hidden layer. The sonar benchmark training set turns to be linearly separable, i.e., it needs no hidden units at all (although back-prop training without any hidden units almost always fails to produce a successful solution).

For the two class distinction problem, networks with various number of hidden units $(1,2,3,4,6,8,10,12, \ldots$ etc.) were trained with normal gradient descent as well as a modified version that specifically targets enhanced fault tolerance (the details of the modified algorithm are presented in subsection 2.1). Each network was then replicated several times and the resulting PFT was evaluated under the single fault assumption (i.e., only a single link or unit can fail). For links we considered 3 types of faults: stuck-at \pm MAX and 0 where MAX is the largest weight magnitude in the trained network. For each hidden unit we considered two types of faults: it's output stuck at \pm Output_Max. For a unit, stuck-at-0 fault on the output is not necessary: if the other two faults can be tolerated, then a stuck-at- 0 fault on the output of that unit can also be tolerated.
The PFT is simply the fraction of outputs that remain correct when all single faults are considered. To generate this value, every weight and unit in the seed network is set stuck at each fault type. For each fault, all I/O patterns are applied and the number of outputs that remain correct are accumulated. This total is divided by the total number of cases, which equals

[n_outputs $\times$ n_io_patterns $\times$

$$
\left.\left(3 \cdot n_{-} \text {weights }+2 \cdot n_{-} \text {hidden_units }\right)\right]
$$

where " $n \_X$ " denotes "number of $X$ ", for instance, n_hidden_units denotes the number of hidden units). This fraction (PFT) is calculated for different number of replications and plotted as a function of replications as well as a function of the total number of hidden units in the final resulting network. For each size of the seed network (1, $2,3, \ldots$ hidden units), 50 sample networks were generated. For each sample network the PFT was evaluated for increasing number of replications. The average of the 50 PFT values was used to generate the plots illustrated in the next Section (3).

For the two class distinction problem, The PFT was evaluated on training data as well as on the combined set containing the training and test data.

The Sonar benchmark data sets and networks are significantly larger. Hence we did not train these networks for fault tolerance. We experimented with varying number of hidden units and used the resulting networks as seed nets and evaluated the resulting PFT as a function of replications and of the total size of the resulting network. For each value of n_hidden_units in the seed network, 10 sample seed networks were generated and their PFT values were calculated. The numbers plotted in the figures are the averages over these 10 runs.

\section{A. Improved Training Algorithms}

One way to enhance PFT is to explicitly add constraints to the search algorithm and solve the constrained optimization problem. This was the approach taken in 5] where they consider only stuck-at 0 faults on the outputs of the hidden units. For each such fault, they put a constraint that the total error (mean sum squared error over all output units over all patterns) should not increase by more than some small amount, say $\varepsilon$. They then solve the constrained optimization problem for several values of $\varepsilon$ and try to estimate the minimum value of $\varepsilon$ for which the value of the objective function (sum squared error) is near zero. This approach, though analytically well founded, does not guarantee a solution. Also, the search can get stuck at local minima; the solution obtained depends on the starting point; and is not guaranteed to be the best solution. More- 
over, the authors did not test the procedure on a wide variety of examples or benchmarks. They tested the algorithm on only one specific example in which there were a small number of hidden units in a single hidden layer and the size of the training data set was also small.

Another way of approaching constrained optimization is the "penalty function method" [16]. Here, instead of directly solving the constrained problem, the constraints are incorporated in the objective function.

$$
\begin{aligned}
E= & \sum_{p} \sum_{o}\left(t_{o p}-y_{o p}\right)^{2}+ \\
& \alpha \sum_{h} \sum_{f} \sum_{p} \sum_{o}\left(t_{o p}-y_{o p f h}\right)^{2}
\end{aligned}
$$

where

$$
\begin{aligned}
& E= \text { Objective function to be minimized } \\
& t_{o p}, y_{o p}= \begin{array}{l}
\text { target and actual outputs (without } \\
\text { any faults) for output unit } o \\
\text { for pattern } p
\end{array} \\
& y_{\text {opfh }}=\begin{array}{l}
\text { actual output of unit } o \text { for pattern } p, \\
\text { upon a fault of type } f \text { on the output } \\
\text { of hidden unit } h \\
\text { weight of the extra terms relative to }
\end{array} \\
& \text { normal error terms }
\end{aligned}
$$

Note that there are two sets of terms in this objective function $E$. The first summation includes the normal error terms. The second summation accrues the errors that arise due to faults and is the penalty function. Instead of solving a constrained problem, the error arising as a result of faults has been included in the objective function itself, thereby "penalizing" any deviations from target outputs upon faults. When $E$ is minimized, the error between target outputs and the network outputs upon faults is also minimized. Thus the addition of these terms forces the search algorithm to look for a solution with better fault tolerance. These terms in the objective function that encourage fault tolerance are henceforth referred to as "extra terms". The (nonnegative) parameter $\alpha$ is the weight of the penalty function (or the penalty) relative to the normal error terms. Typically, $\alpha<1$. By suitably adjusting $\alpha$, one can obtain different solutions.

We consider stuck-at $\pm M A X$ faults on the output of each hidden unit, where $M A X$ is the maximum magnitude which the output of a hidden unit can assume. The number of extra terms can be prohibitively large if fault constraints are applied on a per-link basis. For instance, In the Sonar benchmark there are 60 input units. If the number of hidden units is 50 , the number connections is about 3000. Here, it is practically infeasible to consider all faults for each weight in the training algorithm. Moreover, any faults affecting weights or bias associated with a hidden unit can only affect the rest of the net through the output of that unit. Hence, considering the worst cases, (viz., the output of each hidden unit stuck-at $\pm M A X$ ) should suffice. In other words, if stuck-at $\pm M A X$ faults on the output of a hidden unit can be tolerated, then any fault affecting the weights or bias of that unit can also be tolerated.

Note that this restriction is applied only during training with the improved algorithm to keep the simulation time manageable. The PFT evaluation includes all faults on every link as well as each hidden unit.

The computations are slightly different from those performed in the normal back propagation method. In particular, the increments in the weights are now calculated as a sum of two parts, one due to the normal error terms and one due to the extra (fault tolerance) terms:

$$
\Delta w_{i j}^{\text {total }}=\Delta w_{i j}^{\text {normal_terms }}+\alpha \cdot \Delta w_{i j}^{\text {extra」terms }}
$$

Different learning rates and momentum coefficients are used for the normal and the extra terms.

\section{Results}

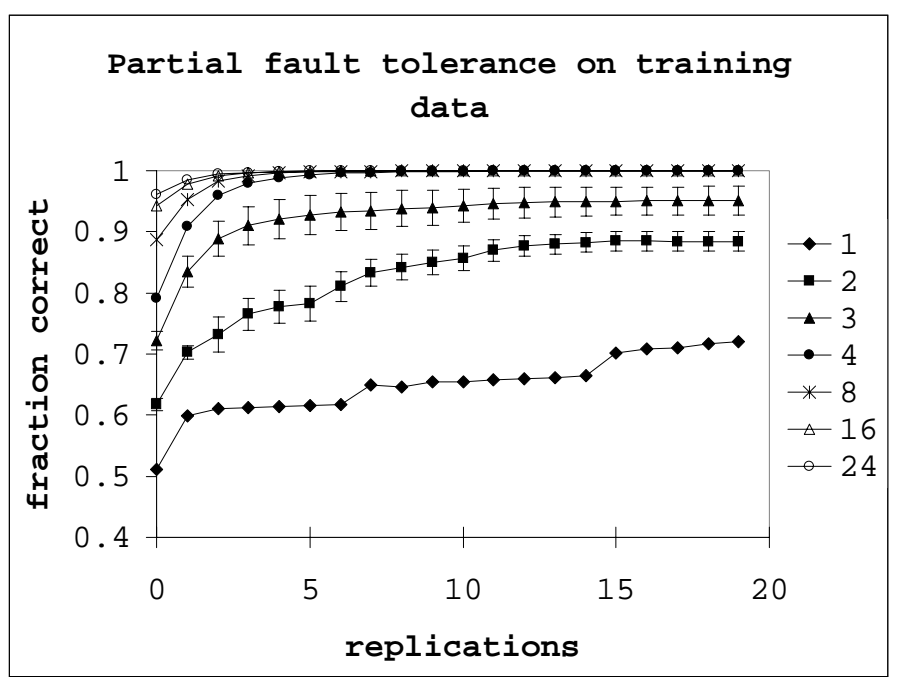

Fig. 2

THE TWO-CLASS DISTINCTION PROBLEM: PFT ON

TRAINING DATA AS A FUNCTION OF THE NUMBER OF REPLICATIONS FOR DIFFERENT SIZED SEED NETWORKS.

Figure 2 illustrates the PFT of networks trained on the 2-class distinction problem. The seed network size was varied from 1 hidden unit to 24 hidden units. Each point is the average of 50 runs. The bars around each point represent the confidence interval for $\alpha=99 \%$ (the confidence intervals were generated for every point on every plot, but are omitted from most plots for the sake of clarity: the confidence bars would have cluttered the diagram). Fig- 
ure 3 illustrates the PFT as a function of the total number of units in a network. Here, the PFT was evaluated only

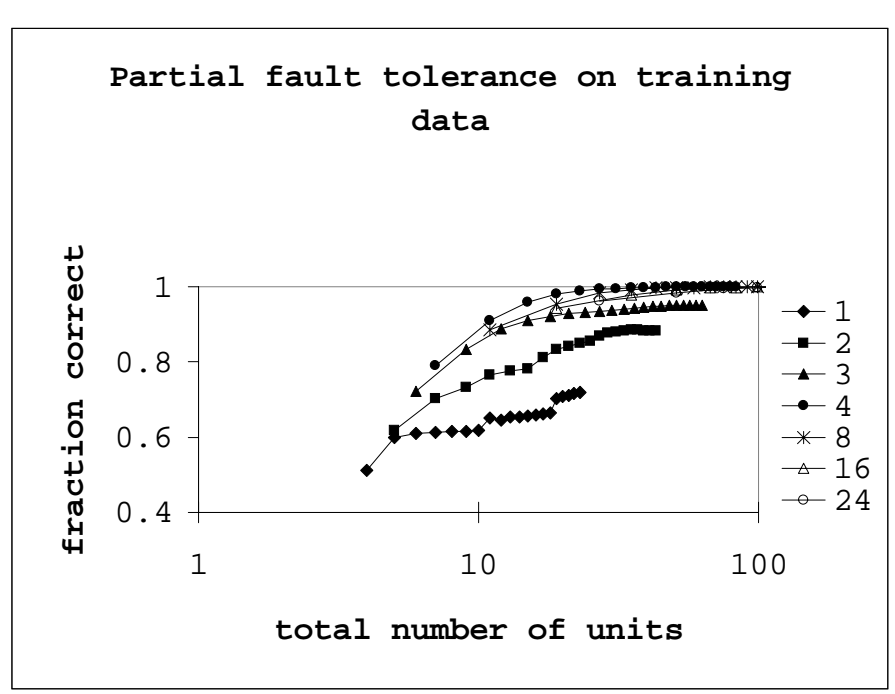

Fig. 3

THE TWO-CLASS DISTINCTION PROBLEM: PFT ON TRAINING DATA AS A FUNCTION OF THE TOTAL NUMBER OF UNITS FOR DIFFERENT SIZED SEED NETWORKS.

on the training data. Figure 4 shows the PFT evaluated on combined training and test data, as a function of the total number of units. Figure 5 zooms in on the interesting part of Figure 4.

The data indicates the following trends:

(1) As the size of the seed network (which is replicated) is increased (from the minimal), the initial PFT (without any replications) improves. The improvement tapers off as the size of the initial network is further increased.

(2) The maximum PFT enhancement occurs due to the first few (about 5 or so) replications. This is more clearly exhibited by the seed networks with larger sizes.

(3) More interesting: for the same total number of units, replicating larger-than-minimal networks leads to higher PFT. For instance, in Figure 4, for any value of total number of units (i.e., any abscissa) the PFT curve generated by the seed network of size 4 is higher than the corresponding curves generated by a seed networks of sizes 2 and 3 .

(4) However, as the size of the seed network is increased further, there is a saturation of sorts in this PFT enhancement with the size of the seed network. In fact when the size of the seed network grows beyond a certain threshold, the plots cross each other, thereby indicating two things:

(i) Replicating a smaller network yields higher PFT than training the same sized network with enhanced algorithm. For example, the initial PFT of seed networks of sizes 16 and 24 in Figure [5] is lower than the network of the

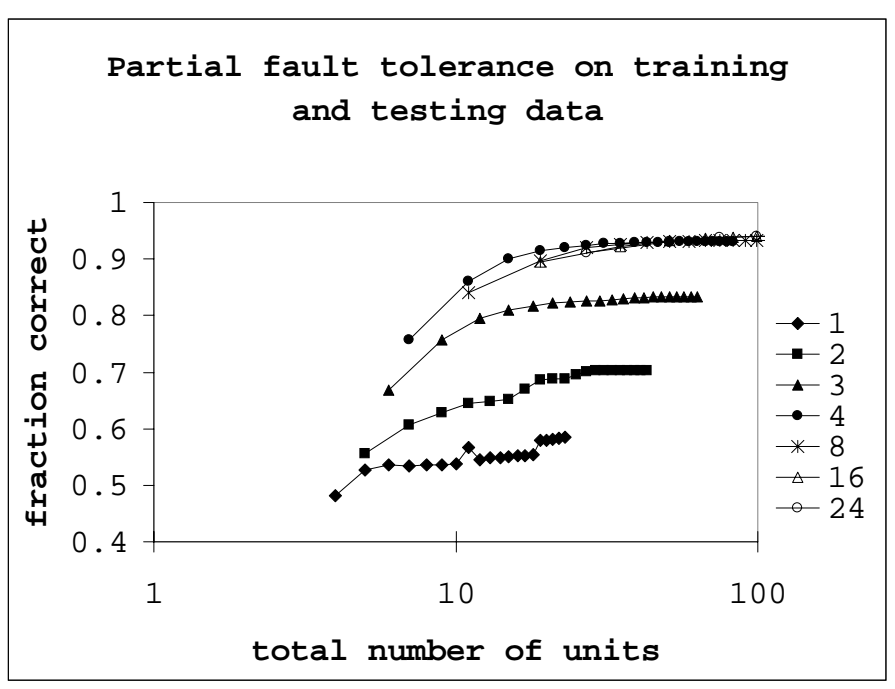

Fig. 4

THE TWO-CLASS DISTINCTION PROBLEM: PFT ON COMBINED TRAINING AND TEST DATA AS A FUNCTION OF THE TOTAL NUMBER OF UNITS FOR DIFFERENT SIZED SEED NETWORKS.

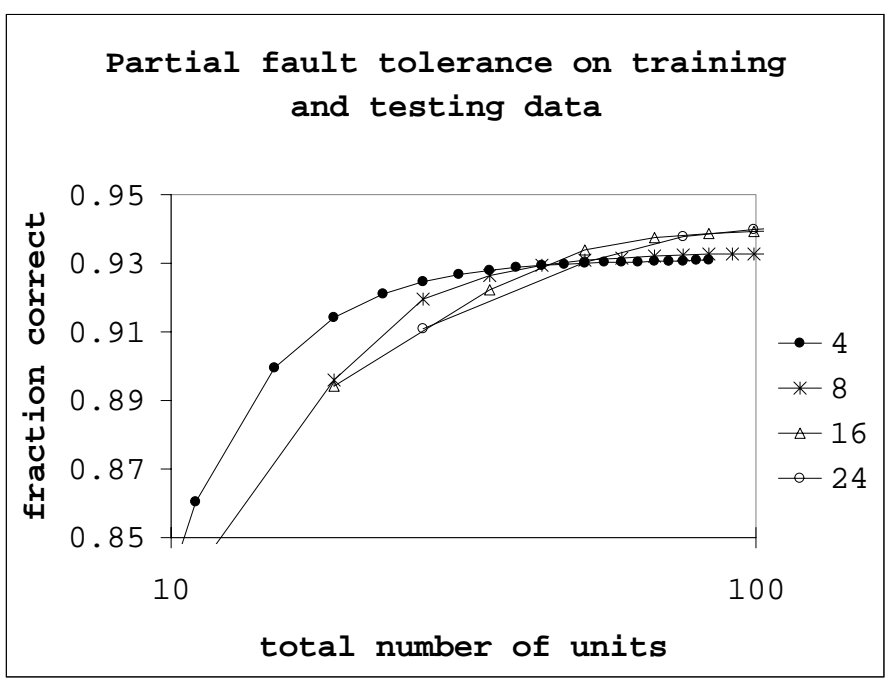

Fig. 5

THE TWO-CLASS DISTINCTION PROBLEM: SAME AS PREVIOUS FIGURE, DIFFERENT $x$ AND $y$ AXES INTERVALS ARE SELECTED TO ZOOM-IN ON THE REGION OF INTEREST. 
same size obtained by replicating seed network(s) of size 8 which is in turn lower than PFT obtained by replicating seed networks of size 4 .

(ii) For a given final size, there is an optimal sized seed network. For example, say the final size 24 hidden units. This could be achieved by replicating a seed network of 2 units 12 times, a seed network of 4 units 6 times, a seed network of 8 units 3 times or a training a network of 24 units without replications. As Figure 5 illustrates, the highest PFT (for a final size $=24$ hidden units) is obtained by replicating a seed network of 4 units 6 times. Thus the optimal is in the middle (neither at the two extremes viz., replicate the minimal network of size 2, 12 times; nor train the full network of size 24 without any replications).

Figure 6 illustrates the effect of using seed networks

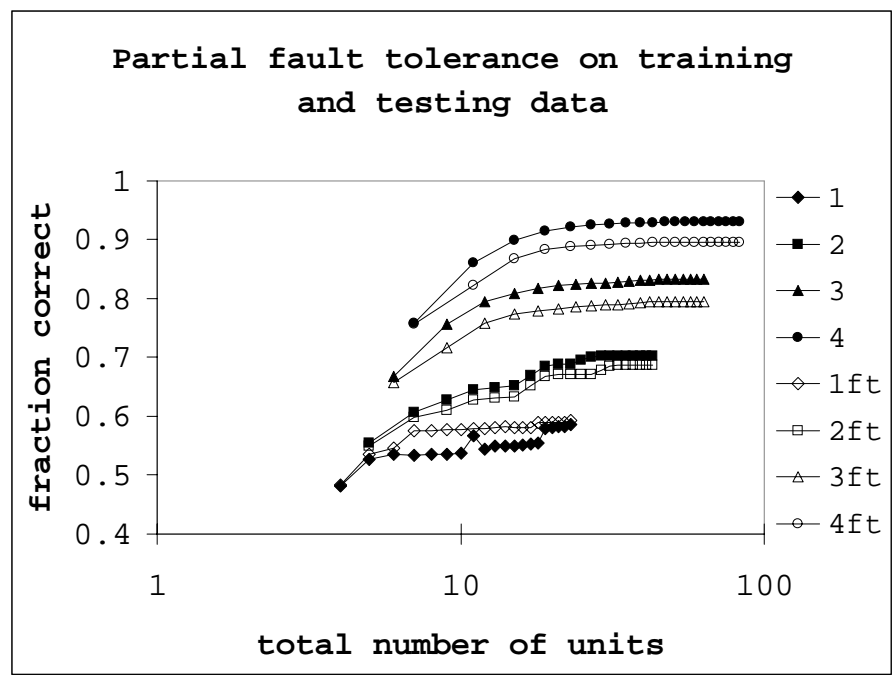

Fig. 6

THE TWO-CLASS DISTINCTION PROBLEM: PFT ON COMBINED TRAINING AND TEST DATA AS A FUNCTION OF THE TOTAL NUMBER OF UNITS FOR DIFFERENT SIZED SEED NETWORKS TRAINED WITH (A) NORMAL TRAINING AND

(B) FAULT TOLERANCE-ENHANCEMENT TRAINING

trained with the enhanced algorithm. These show higher initial PFT (without replications) on the training data than the corresponding networks trained by normal algorithm as expected. However when the PFT is evaluated on the combined training and test data, it is found to be lower than that of nets which were not trained for fault tolerance.

The same trends are observed for the sonar benchmark as illustrated by Figures 7 - 8 .

\section{Discussion and conclusions}

The above observations can be explained as follows. When the network has the minimal size required to suc- cessfully learn a task, the outputs belong to the correct class, but perhaps barely so. For the sake of illustration, assume that units are sigmoidal with outputs in the range $[-0.5,+0.5]$ and an output above 0.25 is deemed to represent a "logical high" or "1" while an output below -0.25 is deemed to be a logical "low" or " 0 ". Then when the network is of minimal capacity, it's outputs might be just barely in the right logical classes. This seems intuitive

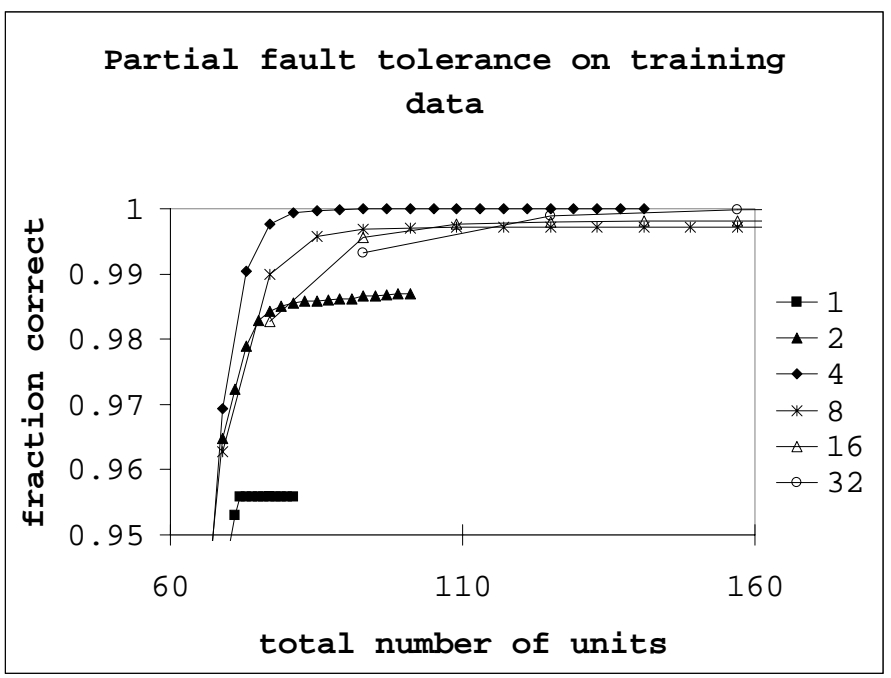

Fig. 7

THE Sonar benchmark [14], [15] : PFT ON TRAINING DATA AS A FUNCTION OF THE TOTAL NUMBER OF UNITS FOR DIFFERENT SIZED SEED NETWORKS.

because the network has barely enough capacity to learn the task.

On the other hand, a network with more capacity is more likely to produce "cleaner" outputs (closer to the desired values of \pm 0.5 ). Due to the nature of the replication procedure, the cleaner the outputs of the seed network, the fewer is the number of replications required [6], [8], [10]. Hence larger-than-minimal seed networks are likely to need fewer replications. The interesting point is that depending on the initial size, fewer replications of the larger-than-minimal network typically yield higher PFT (for the same final size) than a large number of replications of the minimal network.

It is clear that beyond a certain threshold driving the outputs closer to \pm 0.5 (i.e., making them "cleaner") begins to require a large number of units because the sigmoid function approaches its extremes asymptotically. Thus, when the size of the seed network is beyond a certain threshold, the added "closeness" to correct outputs is marginal (thereby implying that the number of replications required to achieve a certain level of PFT is about the same) but the 


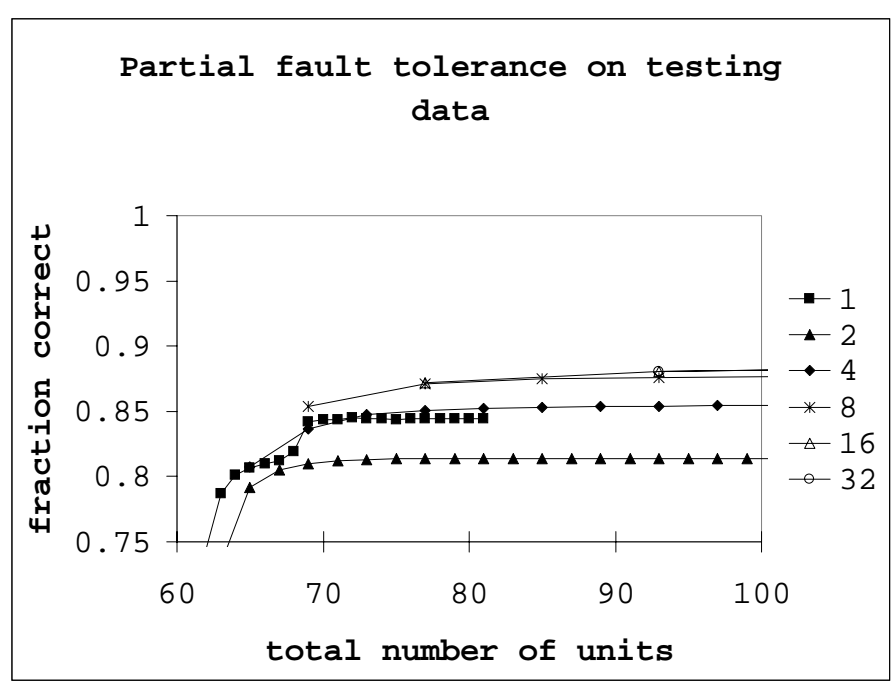

Fig. 8

THE Sonar benchmark : PFT ON TEST DATA AS A FUNCTION OF THE TOTAL NUMBER OF UNITS FOR DIFFERENT SIZED SEED NETWORKS.

size of the seed network is large, so this is not the optimum way to achieve high PFT. This explains the second part of the phenomenon, viz., for a given fixed final size, there is a seed network of optimal size, increasing the seed network size beyond this threshold is a losing proposition.

When nets are trained by the enhanced algorithm, it is in a sense equivalent to constrained optimization. This is likely to cause the outputs to not go to extremes (the same capacity is now being used to do other things as well, besides conquering the training set). In other words, the outputs are likely to be less "clean" or close to the desired levels. Hence the number of replications required is higher as seen in the data.

In conclusion, we have shown that in order to enhance PFT by replication, the seed network should have its optimal size which is not at either extreme (minimal or very large size). We explained this behavior by qualitative arguments. Moreover, we have further corroborated our earlier findings, viz., brute-force method of replication is better at improving PFT than using enhanced algorithms without replications. The importance of improved training algorithms, however, cannot be underestimated. In most practical situations, the large redundancy required by the replication scheme is not likely to be affordable. In such cases there is no alternative but to use an improved training algorithm that utilizes every available parameter to the fullest possible extent.
[1] C. H. Sequin and R. D. Clay, "Fault Tolerance in Artificial Neural Networks," in Proc. of Int. Joint Conf. on Neural Nets (IJCNN), San Diego, CA, Jun. 1990, vol. I, pp. 703-708.

[2] T. Petsche and B. W. Dickinson, "Trellis Codes, Receptive Fields, and Fault Tolerant, Self-Repairing Neural Networks," IEEE Transactions on Neural Networks, vol. 1, no. 3, pp. 154-166, Jun. 1990.

[3] B. E. Segee and M. J. Carter, "Fault Tolerance of Pruned Multilayer Networks," in Proc. of Int. Joint Conf. on Neural Nets (IJCNN), Seattle, WA, 1991, pp. 447-452.

[4] B. E. Segee and M. J. Carter, "Comparative Fault Tolerance of Parallel Distributed Processing Networks," IEEE Transactions on Computers, vol. 43, Nov 1994.

[5] C. Neti, M. H. Schneider, and E. D. Young, "Maximally Fault Tolerant Neural Networks," IEEE Transactions on Neural Networks, vol. 3, no. 1, pp. 14-23, Jan. 1992.

[6] D. S. Phatak and I. Koren, "Fault Tolerance of Feedforward Neural Nets for Classification Tasks," in Proceedings of International Joint Conference on Neural Nets (IJCNN), Baltimore, MD, Jun. 1992, vol. II, pp. 386-391.

[7] P. W. Protzel, D. L. Palumbo, and M. K. Arras, "Performance and Fault Tolerance of Neural Networks for Optimization," IEEE Transactions on Neural Networks, vol. 4, no. 4, pp. 600-614, July 1993.

[8] D. S. Phatak, Fault Tolerance of Feed-Forward Artificial Neural Nets and Synthesis of Robust Nets, Ph.D. thesis, University of Massachusetts, Amherst, 1994.

[9] A. F. Murray and P. J. Edwards, "Enhanced MLP performance and fault tolerance resulting from synaptic weight noise during training," IEEE Transactions on Neural Networks, vol. 5, no. 5, pp. 792-802, Sept. 1994.

[10] D. S. Phatak and I. Koren, "Complete and Partial Fault Tolerance of Feedforward Neural Nets," IEEE Transactions on Neural Networks, vol. 6, no. 2, pp. 446-456, Mar. 1995.

[11] C. M. Bishop, "Training With Noise is Equivalent to Tikhonov Regularization," Neural Computation, vol. 5, no. 1, pp. 108-116, Jan 1995.

[12] Dhananjay S. Phatak, "Fault Tolerant Artificial Neural Networks," in Proceedings of the 5th IEEE Dual Use Technologies and Applications Conference, Utica/Rome, May 1995, pp. 193 198.

[13] Dhananjay S. Phatak, "Relationship between fault tolerance, generalization and the Vapnik-Chervonenkis (VC) dimension of feedforward ANNs.," in International Joint Conference on Neural Networks, Washington, DC, July 1999, manuscript \# 272 on the CD-ROM proceedings (session 3.7).

[14] S. E. Fahlman et. al., Neural Nets Learning Algorithms and Benchmarks Database, maintained by S. E. Fahlman et. al. at the Computer Science Dept., Carnegie Mellon University.

[15] R. P. Gorman and T. J. Sejnowski, "Analysis of Hidden Units in a Layered Network Trained to Classify Sonar Targets," Neural Networks, vol. 1, pp. 75-89, 1988.

[16] D. G. Luenberger, Linear and Nonlinear Programming, AddisonWesley Publishing Company, Reading, Massachusetts, 1984.

\section{References}

